
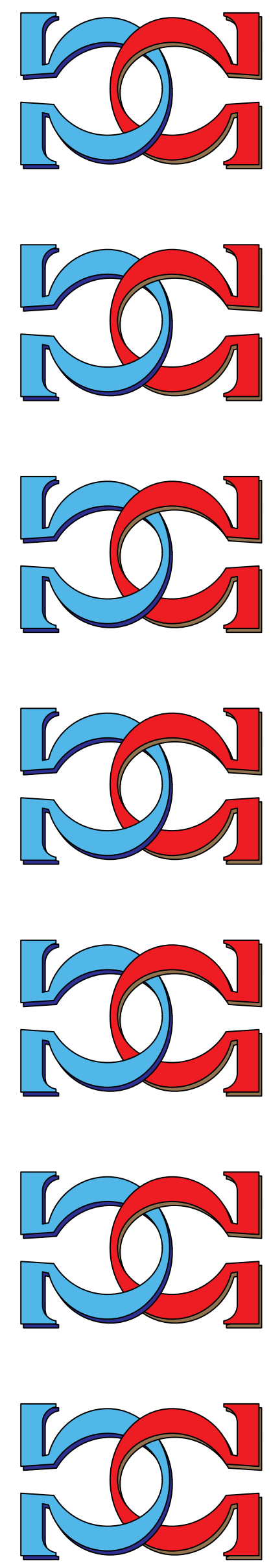

\section{CDMTCS}

\section{Research}

\section{Report}

\section{Series}

\section{Exact results on manipulability of positional voting rules}

\section{Geoffrey Pritchard}

Department of Statistics, University of Auckland, Auckland, New Zealand

Mark C. Wilson

Department of Computer Science, University of Auckland, Auckland, New Zealand

CDMTCS-274

December 2005

Centre for Discrete Mathematics and

Theoretical Computer Science 


\title{
Exact results on manipulability of positional voting rules
}

\author{
GEOFFREY PRITCHARD \\ Department of Statistics \\ The University of Auckland, Private Bag 92019 \\ Auckland, New Zealand \\ geoffestat.auckland.ac.nz \\ MARK C. WILSON \\ Department of Computer Science \\ The University of Auckland, Private Bag 92019 \\ Auckland, New Zealand \\ mcw@cs.auckland.ac.nz
}

December 13, 2005

\begin{abstract}
We consider 3-candidate elections under a general scoring rule and derive precise conditions for a given voting situation to be strategically manipulable by a given coalition of voters. We present an algorithm that makes use of these conditions to compute the minimum size $M$ of a manipulating coalition for a given voting situation.

The algorithm works for any voter preference model - here we present numerical results for IC and for IAC, for a selection of scoring rules, and for numbers of voters up to 150. A full description of the distribution of $M$ is obtained, generalizing all previous work on the topic.

The results obtained show interesting phenomena and suggest several conjectures. In particular we see that rules "between plurality and Borda" behave very differently from those "between Borda and antiplurality".
\end{abstract}

\section{Introduction}

Following the proofs by Gibbard [5] and Satterthwaite [15] that every reasonable voting rule can be manipulated, several authors have tried to quantify the probability of such an event, under various assumptions on the distribution of voter opinions. These papers have differences in: the definition of manipulability; the measure of manipulability; the assumptions on voter preferences; whether the answers sought are exact (either derived via numerical computation or via an analytic formula for a fixed number of voters) or asymptotic (as the number of voters grows large). They also differ markedly in their generality and mathematical sophistication.

The present article considers in detail the problem of coalitional manipulation of a positional voting rule. Our aims throughout are generality, simplicity and rigour. We consider a general positional voting rule, derive linear equations and inequalities describing exactly whether or not a given voting situation is manipulable by a given subset of the voters, and use these to show how to efficiently compute the minimum size of a manipulating coalition. We present numerical results based on enumeration of the manipulable voting situations, for positional voting rules in the 3 -alternative case. This allows a complete picture of the distribution of the size of a minimum manipulating coalition for moderate numbers of voters, and suggests various conjectures about the asymptotic behaviour. 
The layout of the paper is as follows. In the remainder of this section, we outline in detail our basic assumptions and definitions. In Section 2 we derive the linear systems mentioned above, and solve them analytically as far as possible. In Section 3 we describe our enumeration algorithm and its implementation. In Section 4 we present a selection of interesting numerical results obtained using our program. In Section 5 we further discuss the significance of these results and discuss the relationship between our work and that of other authors. Finally, in Section 6 we discuss possible extensions and future work.

Impatient readers may wish to jump straight to the linear conditions in Figure 1, read Theorem 2.5, peruse the tables and graphs in Section 4 and then go on to the open problems in Section 6.

\subsection{Basic definitions}

We suppose that there are $m$ alternatives or candidates $a_{1}, \ldots, a_{m}$ for an election. We deal fully only with the case of $m=3$ alternatives, but much of our methodology carries over to general $m$ (however when $m \geq 4$, exhaustive computation of exact results rapidly becomes infeasible for even moderate values of $n$ ). We usually write $a, b, c$ instead of $a_{1}, a_{2}, a_{3}$.

Definition 1.1. A profile is specified by giving an opinion or preference (a linear ordering of the alternatives) for each voter.

There are $M=m$ ! different possible opinions, which in our case we order lexicographically in the usual way: for $m=3$ this is $a b c, a c b, b a c, b c a, c a b, c b a$.

Definition 1.2. The above ordering of opinions induces a partition of the set $\mathcal{V}$ of voters into subsets $\mathcal{V}_{1}, \ldots, \mathcal{V}_{M}$. We write $\nu_{i}$ for the cardinality of $\mathcal{V}_{i}$. For each subset $X \subseteq \mathcal{V}$, the above partition of $\mathcal{V}$ induces a corresponding partition of $X$, and we write $x_{i}$ for the cardinality of $X_{i}:=\mathcal{V} \cap X$. The $M$-tuple $\left(\nu_{1}, \ldots, \nu_{M}\right)$ is called the voting situation corresponding to the voters' preferences. The set of all possible voting situations we denote by $\mathcal{S}$.

Remark 1.3. The cardinality of $\mathcal{S}$ is given by the binomial coefficient $\left(\begin{array}{c}n+M-1 \\ M-1\end{array}\right)$, where $n=|\mathcal{V}|$ is the number of voters. For $m=3$ this number is asymptotically of order $n^{5}$, whereas the number of profiles is $n$ !.

For voting rules which are anonymous (invariant under all permutations of the set $\mathcal{V}$ of voters), we need only consider voting situations, not profiles. We shall do this throughout.

Definition 1.4. A positional or scoring rule is defined for a given $m$ by a real $m$-tuple $w$ whose entries are in (not necessarily strictly) decreasing order. Alternative $k$ receives score $w_{i}$ from a voter $v$ if and only if $k$ is in position $i$ of $v$ 's preference order. The total score $|k|$ of $k$ is obtained by summing the scores given to $k$ by each voter. The alternative with highest total score wins.

Definition 1.5. The scoreboard associated to a voting situation $\sigma$ is the $m$-tuple of scores $\left(\left|a_{1}\right|, \ldots,\left|a_{m}\right|\right)$. We denote by $\alpha$ the score map sending an element of $\mathcal{S}$ to the associated scoreboard.

Remark 1.6. In the case $m=3$ we have

$$
\begin{aligned}
& |a|=w_{1}\left(\nu_{1}+\nu_{2}\right)+w_{2}\left(\nu_{3}+\nu_{5}\right)+w_{3}\left(\nu_{4}+\nu_{6}\right) ; \\
& |b|=w_{1}\left(\nu_{3}+\nu_{4}\right)+w_{2}\left(\nu_{1}+\nu_{6}\right)+w_{3}\left(\nu_{5}+\nu_{2}\right) ; \\
& |c|=w_{1}\left(\nu_{5}+\nu_{6}\right)+w_{2}\left(\nu_{2}+\nu_{4}\right)+w_{3}\left(\nu_{1}+\nu_{3}\right) .
\end{aligned}
$$




\subsection{Tie-breaking for positional rules}

All positional rules contain the possibility of tied scores for first place. In this event, a separate tiebreaking rule will be needed. One common tie-breaking rule is to resort to a pre-determined arbitrary order (for example, lexicographic order, or the French practice of allowing the oldest candidate to prevail). Though simple to implement, this is not always so convenient for analysis. Another method is to give one of the voters a casting vote. However, this is even less tractable from a theoretical point of view, as it means that the winner can no longer always be determined from the voting situation only, but may require consultation of the full profile.

Here we will use the random tie-breaking method. That is, in the event of a tie, the winner will be chosen at random, with all candidates with the maximum score being equally likely to be chosen. This method complicates the voting by introducing probabilistic considerations, and of course does not yield a voting rule in the classical deterministic sense. However, it has the great advantage that it preserves the symmetry among candidates (such rules are called neutral). This is important for the large-scale computational studies undertaken in this paper, as the symmetry can be exploited to reduce the amount of work required. Furthermore, it can be strongly argued that such a tie-breaking method is fairer than any method that breaks the symmetry between candidates.

\subsection{Manipulation}

We present below the basic definitions used throughout this article. There are many other concepts of manipulability; we discuss this further in Section 5.

Definition 1.7. We define manipulability of a voting situation in stepwise fashion as follows.

- A rule is manipulable at a voting situation $\sigma$ if and only if there is exists a profile $\pi$ giving rise to $\sigma$, at which the rule is manipulable;

- a rule is manipulable at the profile $\pi$ if and only if there exists a manipulating coalition at this profile;

- a subset $X \subseteq \mathcal{V}$ is a manipulating coalition at the profile $\pi$ if and only if there is a profile $\pi^{\prime} \neq \pi$ which agrees with $\pi$ on $\mathcal{V} \backslash X$ and is preferred to $\pi$ by all members of $X$;

- $\quad$ - a profile $\pi^{\prime}$ with (untied) winning candidate $a^{\prime}$ is preferred by voter $v$ to a profile $\pi$ with (untied) winning candidate $a$ if $v$ prefers $a^{\prime}$ to $a$;

- To compare profiles in which scores are tied for first place, it is necessary to compare probability distributions on the set of candidates. We do this in the standard sense of stochastic order. That is, $v$ prefers $\pi^{\prime}$ to $\pi$ if for each $k=1, \ldots, m$ the probability of electing one of his most-favoured $k$ candidates under $\pi^{\prime}$ is no less than under $\pi$. (If $\pi^{\prime} \neq \pi$, the condition implies that this probability will be strictly greater for some $k$.)

Note in particular that a voter will never prefer to increase the probability of electing the candidate he favours least, or to decrease the probability of electing the candidate he favours most. In three-candidate elections, these two conditions constitute a full description of the preference rule.

Example 1.8 (manipulation). Consider the plurality rule defined by the weight vector $(1,0,0)$, and an election in which sincere preferences are such that 4 voters have the opinion $a b c, 3$ have the opinion $b c a$, and 2 have the opinion $c a b$. The scoreboard is then $(4,3,2)$ and $a$ is the sincere winner.

The subset consisting of all the $b c a$ and $c a b$ voters can manipulate. Indeed, if the bca voters vote strategically as $c b a$ and the $c a b$ voters continue to vote $c a b$, then the original winner $a$ is replaced by $c$. Each of the types of voters in the coalition prefers the new election outcome to the sincere one. 


\begin{tabular}{|l|l|c|c|c|}
\hline Sincere outcome & Manipulated outcome & Possible? & Coalition member types & Type \\
\hline $1 .|a|>|b| \geq|c|$ & (i) $b$ wins & yes & $b a c, b c a, c b a(\operatorname{prefer} b$ to $a$ ) & I \\
& (ii) $a, b$ tie & yes & $b a c, b c a, c b a(\operatorname{prefer} b$ to $a)$ & I \\
& (iii) $c$ wins & yes & $c a b, c b a, b c a(\operatorname{prefer} c$ to $a$ ) & I \\
& (iv) $a, c$ tie & yes & $c a b, c b a, b c a(\operatorname{prefer} c$ to $a$ ) & I \\
& (v) $b, c$ tie & no & & \\
& (vi) 3 -way tie & no & & \\
\hline $2 .|a|=|b|>|c|$ & (i) $a$ wins & yes & $a b c, a c b, c a b($ prefer $a$ to $b)$ & II \\
& (ii) $b$ wins & yes & $b a c, b c a, c b a($ prefer $b$ to $a$ ) & II \\
& (iii) $c$ wins & no & & \\
& (iv) $a, c$ tie & yes & $c b a, c a b, a c b(\operatorname{prefer} c$ to $b$ ) & III \\
& (v) $b, c$ tie & yes & $c a b, c b a, b c a(\operatorname{prefer} c$ to $a$ ) & III \\
& (vi) 3 -way tie & no & & \\
\hline 3. $|a|=|b|=|c|$ & any & no & & \\
\hline
\end{tabular}

Table 1: Manipulation of 3-candidate elections.

Note that no manipulation in favour of $b$ is possible in this situation, since the only voters preferring $b$ to $a$ are already contributing the maximum score to $b$ and the minimum to $a$.

Example 1.9 (manipulation involving ties). Consider the Borda rule, defined by the weight vector $(2,1,0)$, and an election in which sincere preferences are such that 1 voter has the opinion $a b c, 3$ voters have the opinion $a c b, 4$ voters have opinion $b a c$, and 3 voters have opinion $c b a$. The scoreboard is then $(12,12,9)$.

The subset consisting of all $a c b$ and $c b a$ voters can manipulate. If all of them change their votes to $c a b$, then the new scoreboard becomes $(12,9,12)$. Note that all members of the coalition prefer $c$ to $b$ and hence prefer this election outcome in the sense of stochastic order. The winning probability distribution on the candidates has changed from $(1 / 2,1 / 2,0)$ to $(1 / 2,0,1 / 2)$.

Example 1.10 (minimal manipulation). Consider the rule with weight vector $(4,3,0)$ and the voting situation in which the opinion $a c b$ is held by 1 voter, and the opinions $a b c, b a c, c a b$, and $c b a$ are each held by 2 voters. The scoreboard is $(24,20,19)$. A manipulating coalition can be formed by the two $c b a$ voters and one of the $b a c$ voters (who all change their vote to $b c a$, thus handing victory to $b$ ). The reader may verify that this coalition is minimal, that is, that no coalition with fewer than three members can manipulate. (A coalition of one $c b a$ and one $b a c$ could achieve a 3-way tie, but this is not a preferred outcome for the $b a c$ voter.)

\section{Manipulation of scoring rules in the 3 -alternative case}

For a fixed scoring rule defined by its weight vector $w$, a fixed voting situation $\sigma$, and a fixed subset $X \subseteq \mathcal{V}$, we shall determine whether $X$ is a valid manipulating coalition with the power to change the outcome of the election. Without loss of generality, the voting situation $\sigma$ is such that the candidates' scores are in non-decreasing order: $|a| \geq|b| \geq|c|$. The possible types of manipulations are itemized in Table 1.

We note that there are three essentially different kinds of manipulation possible. In cases 1(i), 1(ii), 1(iii), and 1(iv), there is a clear winner, and manipulation is in favour of one of the other candidates, who is promoted to clear or joint winner. These manipulations we call Type I. In cases 2(i) and 2(ii), there is a tie for first place, and one of the tied candidates is promoted to sole winner by manipulation. 
These we call Type II manipulations. In cases 2(iv), 2(v), there is a tie for first place, and the bottom candidate is promoted to tie with one of the original winners. These we call Type III manipulations.

As noted in Table 1, some types of manipulations never occur. We deal with these first.

Theorem 2.1. For a 3-candidate positional voting rule, no valid coalition can ever manipulate the outcome in any of the following ways.

(i) If $a$ is the sincere winner: creation of a tie between the other two candidates $b$ and $c$.

(ii) If the sincere outcome is a tie between $a$ and b: promotion of the third candidate $c$ to the status of sole winner.

(iii) If the sincere outcome is a tie between all three candidates: creation of any other outcome.

(iv) Under any circumstances: creation of a tie between all three candidates.

Proof. Suppose that $a$ is the sincere winner. A coalition to create a tie between $b$ and $c$ must consist entirely of voters who prefer both $b$ and $c$ to $a$ (types $b c a$ and $c b a$ ). Other voter types will not prefer the $b, c$ tie to a clear win for $a$ in the sense of stochastic order, because it has a higher probability of electing their least-favoured candidate. These voters are already contributing $w_{3}$ (the minimum possible) to the score of $a$, so no strategic voting by them can decrease the score of $a$. Similarly, they are already contributing $\left(w_{1}+w_{2}\right) / 2$ (the maximum possible) to the average score of $b$ and $c$, so no strategic voting by them can increase that average score. Hence, they are unable to bring about any situation in which the average score of $b$ and $c$ exceeds the score of $a$. In particular, the scores of $b$ and $c$ cannot be made equal and greater than $a$ 's score.

The other cases are handled by similar arguments. For (ii), the only possible coalition members are those who prefer $c$ to both $a$ and $b$. For (iii), there are two sub-cases. Making any single candidate the sole winner will be preferred only by voters who rank that candidate first, while creation of any two-way tie will be preferred only by voters who rank the other candidate in last place. For (iv), if there is a sincere sole winner then the 3-way tie will be preferred only by voters who ranked that candidate last. If the sincere outcome is a 2 -way tie, the 3 -way tie will be preferred only by voters who ranked the other candidate first.

\subsection{Linear conditions describing manipulability}

We now turn to the class of possible manipulations described in Table 1. We shall derive linear systems of equations and inequalities that describe exactly which voting situations are manipulable. Each of the types I-III need only be analysed once, since the other cases of the same type are obtained by applying an appropriate transposition of the candidates. We consider the type I case where $|a|>|b| \geq|c|$ and manipulation is in favour of $b$, the type II case $|a|=|b|>|c|$ where manipulation is in favour of $b$, and the type III case $|a|=|b|>|c|$ where manipulation is in favour of $c$.

To save space we shall write $w_{i}-w_{j}$ as $w_{i j}$ from now on. Suppose that a manipulating coalition $X$ contains $x_{i}$ voters of type $i(i=1, \ldots, 6)$. We first consider the type I and type II manipulations - the results are displayed in Figure 1. All members of $X$ must prefer $b$ to $a$, so we have $x_{1}=x_{2}=x_{5}=0$. It is clear that if manipulation is possible at all, it is certainly achievable by each voter ranking $b$ first (this is a dominant strategy for such voters).

Let $y_{1}, y_{2}$ denote the numbers of voters who strategically vote $b a c, b c a$. The strategic scores then 
Figure 1: Integer linear systems describing type I and type II manipulability by a given coalition. The coalition $X$ can manipulate if and only if there exist $y_{1}, y_{2}$ satisfying these conditions.

$$
\begin{aligned}
&|a|-|b| \leq x_{3} w_{21}+x_{4} w_{31}+x_{6} w_{32}+y_{1} w_{12}+y_{2} w_{13} \\
&|c|-|b|<x_{3} w_{31}+x_{4} w_{21}+x_{6} w_{12}+y_{1} w_{13}+y_{2} w_{12} \\
& x_{3}+x_{4}+x_{6}=y_{1}+y_{2} \\
& 0 \leq x_{i} \leq \nu_{i} \text { for } 1 \leq i \leq 6 \\
& 0 \leq y_{1}, \quad 0 \leq y_{2} \\
& \text { all } x_{i}, y_{j} \text { are integers. } \\
& 0<x_{3} w_{21}+x_{4} w_{31}+x_{6} w_{32}+y_{1} w_{12}+y_{2} w_{13} \\
& x_{3}+x_{4}+x_{6}=y_{1}+y_{2} \\
& 0 \leq x_{i} \leq \nu_{i} \text { for } 1 \leq i \leq 6 \\
& 0 \leq y_{1}, \quad 0 \leq y_{2} \\
& \text { all } x_{i}, y_{j} \text { are integers. }
\end{aligned}
$$

become $|a|^{\prime},|b|^{\prime},|c|^{\prime}$, where

$$
\begin{aligned}
& |a|^{\prime}=|a|+\left(y_{1}-x_{3}\right) w_{2}+\left(y_{2}-x_{4}\right) w_{3}-x_{6} w_{3} \\
& |b|^{\prime}=|b|+\left(y_{1}-x_{3}\right) w_{1}+\left(y_{2}-x_{4}\right) w_{1}-x_{6} w_{2} \\
& |c|^{\prime}=|c|-\left(y_{1}-x_{3}\right) w_{3}+\left(y_{2}-x_{4}\right) w_{2}-x_{6} w_{1} .
\end{aligned}
$$

For $b$ to become the sole winner, or tie with $a$, it is necessary and sufficient that the inequalities $|b|^{\prime} \geq$ $|a|^{\prime},|b|^{\prime}>|c|^{\prime}$ be satisfied. We also know that $y_{1}+y_{2}$ equals the size of the coalition. These conditions immediately yield the first system shown in Figure 1. The type II analysis is very similar and yields the second system of Figure 1.

The other cases may be obtained by applying a permutation: $a \leftrightarrow b$ induces the permutation $x_{1} \leftrightarrow$ $x_{3}, x_{2} \leftrightarrow x_{4}, x_{5} \leftrightarrow x_{6}$, while the transposition $b \leftrightarrow c$ induces the permutation $x_{1} \leftrightarrow x_{2}, x_{3} \leftrightarrow$ $x_{5}, x_{4} \leftrightarrow x_{6}$.

The manipulations denoted in Table 1 as "Type III" are more complex. These are rare manipulations in which a tie is replaced with another tie (see Example 1.9). Members of a coalition manipulating in this way have no obvious dominant strategy: since the goal is to match two candidates' scores exactly, any of the six possible candidate orderings could potentially be a useful strategic vote. We shall see below (Theorem 2.3) that for the purposes of this article we may ignore type III manipulations. However, we record the defining linear system in Section 6 for possible future use.

It turns out that we need never consider type III manipulations to determine whether a situation is manipulable, or to compute the minimum size of a manipulating coalition. We now prove this.

Lemma 2.2. If $w=(1,0,0)$ (plurality rule) or $w=(1,1,0)$ (antiplurality rule), type III manipulations are never possible. 
Proof. By symmetry we need only consider the case where the sincere result is $|a|=|b|>|c|$ and we seek to being about a result $|a|=|c|>|b|$. Only voters with opinions $c a b, c b a$ or $a c b$ would prefer the manipulated outcome. First consider plurality. No strategic action by these voters can increase $(|a|+|c|) / 2$ or decrease $|b|$. Similarly in the antiplurality case, none of the willing voters can decrease $(|a|+|b|) / 2$ or increase $|c|$. Thus in no case can $c$ be promoted above $b$ to tie with $a$.

Theorem 2.3. If a voting situation is type III-manipulable by a coalition $X$, then it is also type IImanipulable by a subset of $X$.

Proof. We may assume by Lemma 2.2 that we are dealing with neither plurality nor antiplurality. By symmetry we need only consider the situation when $|a|=|b|>|c|$ and we seek to bring about a tie between $a$ and $c$, with $b$ the clear loser. Suppose that $X$ is a coalition that can manipulate the outcome by means of type III manipulations. We have $x_{1}=x_{3}=x_{4}=0$.

If $x_{6} \neq 0$ then the coalition consisting of a single $c b a$ from $X$ may strategically vote $b c a$, promoting $b$ to sole winner. Similarly if $x_{5} \neq 0$ then a single $c a b$ may vote $a c b$, promoting $a$ to sole winner. Finally, we cannot have $x_{6}=x_{5}=0$, since no strategic action by voters of type $a c b$ can increase $|a|-|b|$.

\subsection{Minimum coalition size}

In the present article, we are chiefly interested in whether a given situation is manipulable by some coalition, and if so, what is the minimum size of a manipulating coalition. As we have seen, these questions may be reduced to integer linear programs of the form

$$
\begin{aligned}
& \min f(x) \\
& \text { s.t. }(x, y) \in R_{2} \\
& \quad x \in \mathbb{Z}^{M}, y \in \mathbb{Z}^{N},
\end{aligned}
$$

where $f(x)=\sum_{i=1}^{M} x_{i}, R_{2}$ is a linear polytope, and for three candidates $M=6$ and $N=2$. (The linear conditions defining $R_{2}$ may include both strict and non-strict inequalities.) The situation is manipulable (in the particular way being considered) if and only if $\mathcal{I}(\mathbb{Z}, \mathbb{Z})$ is feasible; the optimal value of $\mathcal{I}(\mathbb{Z}, \mathbb{Z})$ gives the minimum coalition size.

Exact solution of such problems requires substantial computation in the worst case. Of course, we are dealing with a very small problem. However, several such problems must be solved for each voting situation, so it is important that they be solved as efficiently as possible. We shall now discuss this issue in detail.

Consider $\mathcal{I}(\mathbb{Z}, \mathbb{Z})$. To begin with, define the following simpler problem.

$$
\begin{aligned}
\min & f(x) \\
\text { s.t. } & x \in R_{1} \\
x & \in \mathbb{R}^{M},
\end{aligned}
$$

where $R_{1}=\left\{x \in \mathbb{R}^{M} \mid \exists y \in \mathbb{R}^{N}\right.$ with $\left.(x, y) \in R_{2}\right\}$. Geometrically, $R_{1}$ is the projection of the polytope $R_{2}$ onto the subspace where all $y_{j}$ are zero, and so it is also a linear polytope. The problem $\mathcal{P}(\mathbb{R})$ is thus a linear program; a relatively tractable problem. It is possible to derive $R_{1}$ from $R_{2}$ using the Fourier-Motzkin algorithm [16].

We now list some obvious but useful relations between the integer program $\mathcal{I}(\mathbb{Z}, \mathbb{Z})$ and the projection $\mathcal{P}(\mathbb{R})$. In the Lemma below, note that the optimum of $\mathcal{P}(\mathbb{R})$ may not be attained if there are strict inequalities in the definition of $R_{1}$; if the optimum is attained then its value is of course $m$. 
Lemma 2.4 (relation between $\mathcal{I}(\mathbb{Z}, \mathbb{Z})$ and $\mathcal{P}(\mathbb{R})$ ). The following facts hold.

1. If $\mathcal{P}(\mathbb{R})$ is infeasible, then so is $\mathcal{I}(\mathbb{Z}, \mathbb{Z})$.

2. Suppose that $\mathcal{P}(\mathbb{R})$ and let $m=\inf \left\{f(x): x \in R_{1}\right\}$. Let $(x, y) \in R_{2}$ be an integer point. If either of the following conditions hold, then $(x, y)$ is optimal for $\mathcal{I}(\mathbb{Z}, \mathbb{Z})$.

- $\mathcal{P}(\mathbb{R})$ attains its optimal value and $f(x)=\lceil x\rceil$

- $\mathcal{P}(\mathbb{R})$ does not attain its optimal value and $f(x) \leq m+1$

We now derive explicit descriptions of $R_{1}, R_{2}$. The results are summarized in the following theorem.

Theorem 2.5 (constraints defining $\mathcal{P}$ ). Let $X$ be a manipulating coalition for a given voting situation. Let $x_{i}$ be the number of members of $X$ of type $i$, for $i=1, \ldots, 6$. Then

1. if $|a|>|b| \geq|c|$, then

$$
\begin{aligned}
0 & =x_{1}=x_{2}=x_{5} \\
|a|-|b| & \leq w_{23} x_{3}+w_{12} x_{6} \\
|a|-2|b|+|c| & <3 w_{12} x_{6}
\end{aligned}
$$

or

$$
\begin{aligned}
0 & =x_{1}=x_{2}=x_{3} \\
|a|-|c| & \leq w_{23} x_{5}+w_{12} x_{4} \\
|a|-2|c|+|b| & <3 w_{12} x_{4}
\end{aligned}
$$

(PROJ: Ic)

2. if $|a|=|b|>|c|$, then

$$
\begin{aligned}
& 0=x_{1}=x_{2}=x_{5} \\
& 0<w_{23} x_{3}+w_{12} x_{6}
\end{aligned}
$$

or

$$
\begin{aligned}
& 0=x_{3}=x_{4}=x_{6} \\
& 0<w_{23} x_{1}+w_{12} x_{5}
\end{aligned}
$$

3. it cannot be the case that $|a|=|b|=|c|$.

Proof. We derive the conditions on $x$ in each case by systematically applying Fourier-Motzkin elimination.

Consider the constraints for Case 1(i)/1(ii) displayed in (IP: Ib). First use the equality constraint to eliminate $y_{2}$. This yields the equivalent system

$$
\begin{aligned}
|a|-|b| & \leq w_{23} x_{3}+w_{12} x_{6}+w_{32} y_{1} \\
|c|-|b| & <w_{32} x_{3}+2 w_{12} x_{6}+w_{23} y_{1} \\
0 & \leq x_{3}+x_{4}+x_{6}-y_{1} \\
0 & \leq x_{i} \leq \nu_{i} \text { for } 1 \leq i \leq 6 \\
0 & \leq y_{1} \\
& \text { all } x_{i} \text { and } y_{1} \text { are integers. }
\end{aligned}
$$


We now relax the condition on $y_{1}$ to $y_{1} \in \mathbb{R}$. For each pair of inequalities where the coefficient of $y_{1}$ occurs with different signs, we form a new inequality not involving $y_{1}$ by forming the appropriate positive linear combination of inequalities. This yields

$$
\begin{aligned}
|a|-|b| & \leq w_{23} x_{3}+w_{12} x_{6} \\
|c|-|b| & <w_{23} x_{4}+\left(2 w_{12}+w_{23}\right) x_{6} \\
|a|-2|b|+|c| & <3 w_{12} x_{6} \\
0 & \leq x_{3}+x_{4}+x_{6} \\
0 & \leq x_{i} \leq \nu_{i} \text { for } 1 \leq i \leq 6 \\
& \text { all } x_{i} \text { are integers. }
\end{aligned}
$$

The second and fourth inequalities are redundant since $|b|>|c|$ and all $x_{i}$ are nonnegative. Thus we obtain the stated system (PROJ: Ib). The Case 1(iii)/(iv) conditions are derived similarly and displayed in (PROJ: Ic).

Now consider Case 2(ii), whose integer linear system is displayed in (IP: IIb). A completely analogous argument to the previous case yields the inequalities in (PROJ: IIb). We obtain the equivalent system

$$
\begin{aligned}
0 & <w_{23} x_{3}+w_{12} x_{6}+w_{32} y_{1} \\
|c|-|b| & <w_{32} x_{3}+2 w_{12} x_{6}+w_{23} y_{1} \\
0 & \leq x_{3}+x_{4}+x_{6}-y_{1} \\
0 & \leq x_{i} \leq \nu_{i} \text { for } 1 \leq i \leq 6 \\
0 & \leq y_{1} \\
& \text { all } x_{i} \text { and } y_{1} \text { are integers. }
\end{aligned}
$$

and then the system (PROJ: IIb) by Fourier-Motzkin elimination. Similarly, Case 2(i) yields the system (PROJ: IIa).

The linear programs in Theorem 2.5, along with Lemma 2.4 can be used as a shortcut to solve the integer programs of form $\mathcal{I}(\mathbb{Z}, \mathbb{Z})$. However, caution is required: a feasible point for $\mathcal{P}(\mathbb{R})$, even if it has integral coordinates, need not correspond to any feasible point for $\mathcal{I}(\mathbb{Z}, \mathbb{Z})$. Indeed, it is possible for a solution to $\mathcal{P}(\mathbb{R})$ (with integral coordinates) to exist even when the original problem $\mathcal{I}(\mathbb{Z}, \mathbb{Z})$ is infeasible, as demonstrated by Example 2.6 below. This has apparently not been noticed by previous authors, perhaps because of Theorem 2.9 below, which covers the three most commonly studied rules, namely the plurality, antiplurality and Borda rules.

Example 2.6 $(\mathcal{P}(\mathbb{R})$ feasible but $\mathcal{I}(\mathbb{Z}, \mathbb{Z})$ infeasible). Consider the weight vector $(4,3,0)$ and the voting situation in which 3 voters have sincere preference $b a c, 1$ voter $a c b$, and 1 voter $c a b$. The scores are $|a|=16,|b|=12,|c|=7$. The coalition consisting of the three $b a c$ voters $\left(x_{3}=3\right.$ and all other $x_{i}=0$ ) satisfies the first set of necessary conditions given in Theorem 2.5, suggesting that it might be possible to manipulate in favour of $b$ (Case 1(i)/(ii)). But the necessary and sufficient conditions (IP: Ib) require the existence of integers $y_{1}, y_{2}$ such that

$$
y_{1}+4 y_{2} \geq 7, \quad 4 y_{1}+y_{2}>7, \quad \text { and } y_{1}+y_{2}=3,
$$

which leads to the impossible condition $4 \leq 3 y_{2}<5$. 
Example 2.7 (optimality gap between $\mathcal{I}(\mathbb{Z}, \mathbb{Z})$ and $\mathcal{P}(\mathbb{R})$ ). Consider the voting rule $(8,7,0)$ and the voting situation in which 4 voters have sincere preference $a b c, 1$ voter $a c b, 3$ voters $b a c, 6$ voters $b c a$, and 6 voters $c a b$. The scores are $|a|=103,|b|=100$, and $|c|=97$. Suppose we are interested in Type I manipulations in favour of $c$. Solving the linear program (PROJ: Ic) produces an optimum at the corner $x_{5}=3 / 7, x_{4}=3$. The nearest feasible point with integer coordinates is $x_{5}=1, x_{4}=4$, but this is infeasible for the original problem $(\mathcal{I}(\mathbb{Z}, \mathbb{Z})$ ). Indeed, a coalition of one $c a b$ and four $b c a$ voters cannot manipulate; the best scoreboard they can produce (by all voting $c b a$ ) is $(96,103,101)$, leaving $c$ still in second place.

If a fifth $b c a$ voter is added to the coalition, the scoreboard becomes $(96,102,102)$, but this is not a valid manipulation either, as the new outcome (a $b c$ tie) is not preferred to an outright win for $a$ by the $c a b$ voter. Adding the sixth $b c a$ voter to the coalition produces an outright win for $c$ (scoreboard $(96,101,103))$. This seven-member coalition is not minimal, however. A coalition of the six bca voters may also manipulate, producing the scoreboard $(103,94,103)$ (note that all coalition members prefer the $a c$ tie to an outright win for $a$ ).

The indivisibility of votes plays an important role in this situation. If fractional votes were permitted, only 3.44 votes $(3.01 \mathrm{bca}$ and $0.43 \mathrm{cab}$ ) would need to be changed to produce the scoreboard $(99.99,100,100.01)$ and an outright win for $c$.

Remark 2.8. Example 2.7 shows that the minimum coalition size can be more than the ceiling of the optimum of $\mathcal{P}(\mathbb{R})$.

\subsection{Computing the minimum coalition size}

We now consider the various cases in detail, and simplify the integer programming problems analytically as far as possible. We make liberal use of Lemma 2.4.

First we treat the case $|a|=|b|>|c|$. The relaxation (and hence the original IP) is infeasible if $w_{23} \nu_{3}=0=w_{12} \nu_{6}$. From now on we assume that it is nonempty. Note that $\inf \mathcal{P}(\mathbb{R})=0$.

Suppose first that $w_{12} \nu_{6}>0$. Then optimality is attained at $x_{3}=0, x_{6}=1$, which is the projection of the feasible point $y_{1}=0, y_{2}=1, x_{3}=0=x_{4}, x_{6}=1$ satisfying (IP: IIb).

On the other hand, suppose that $w_{12} \nu_{6}=0$ but $w_{23} \nu_{3}>0$. From (IP: II'b), we see that $-w_{23} x_{3}<$ $w_{23} y_{1}<|b|-|c|-w_{23} x_{3}$ must hold. Since also $y_{1} \geq 0$, this yields the constraint $0<w_{23} x_{3}<|b|-|c|$. If these last inequalities have no integer solution for $x_{3}=1$, the problem is infeasible. Otherwise, we can set $y_{1}=0, y_{2}=1, x_{4}=0, x_{3}=1, x_{6}=0$ in (IP: IIb) and obtain a solution that projects to a feasible point of $\mathcal{P}(\mathbb{R})$, which is optimal as above.

In summary, in this case the minimum size of a coalition that can manipulate in favour of $b$ is 1 when such manipulation is possible. Such manipulation is impossible precisely when $w_{12} \nu_{6}=0$ and either $w_{23}=0$ or $w_{23} \geq|b|-|c|$. A similar argument deals with manipulation in favour of $a$, and we already know that no manipulation in favour of $c$ is possible. Thus the minimum size of a manipulating coalition when $|a|=|b|>|c|$ is 1 , and we have precise conditions in terms of $w$ and the scoreboard for when this is possible.

We now move on to the type I cases, where $|a|>|b|$. We need only consider case 1(i)/(ii), where $b$ but not $c$ has the maximum score after manipulation. That is, the feasible region of $\mathcal{P}(\mathbb{R})$ is given by (PROJ: Ib) and the feasible region of $\mathcal{I}(\mathbb{Z}, \mathbb{Z})$ by (IP: Ib).

If $3 w_{12} \nu_{6} \leq|a|-2|b|+|c|$ or $w_{23} \nu_{3}+w_{12} \nu_{6}<|a|-|b|$, then $\mathcal{P}(\mathbb{R})$ is infeasible and hence so is $\mathcal{I}(\mathbb{Z}, \mathbb{Z})$.

Otherwise, $\mathcal{P}(\mathbb{R})$ is feasible. We may then consider two subcases: $w_{12} \geq w_{23}$ ("easy") and $w_{12} \leq$ $w_{23}$ ("hard"); see Figure 2.3.

First, the easy case. Note that we have $w_{12}>0$. If $w_{12} \nu_{6} \geq|a|-|b|$ then letting $t:=(|a|-|b|) / w_{12}$, the optimal value of $\mathcal{P}(\mathbb{R})$ corresponds to the point $(0, t)$; this optimal value is precisely $t$. By Lemma 


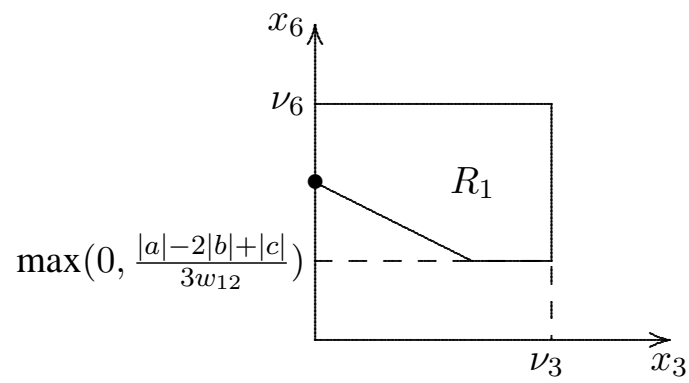

(a) Easy case.

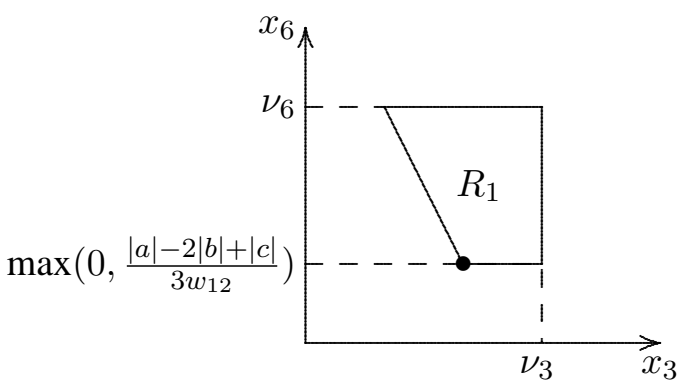

(b) Hard case.

Figure 2: The easy and hard cases of Type I manipulation.

2.4 we see that an optimal solution of $\mathcal{I}(\mathbb{Z}, \mathbb{Z})$ is given by $x_{6}=t=y_{2}$, with all other $x_{i}$ and $y_{j}$ equal to 0 . The optimal value is thus $\lceil t\rceil$. Similarly, if $\nu_{6}<t$ (note that this implies $w_{23}>0$ ), then, letting $s=w_{12}\left(t-\nu_{6}\right) / w_{23}$, the optimal value of $\mathcal{P}(\mathbb{R})$ corresponds to the point $\left(s, \nu_{6}\right)$, and we see by Lemma 2.4 that an optimal solution of $\mathcal{I}(\mathbb{Z}, \mathbb{Z})$ is given by $x_{3}=\lceil s\rceil, x_{6}=\nu_{6}, y_{1}=0$, and $y_{2}=x_{3}+x_{6}$. Note that in this easy case, $\mathcal{I}(\mathbb{Z}, \mathbb{Z})$ is feasible if and only if $\mathcal{P}(\mathbb{R})$ is.

Now for the hard case. If $w_{23} \nu_{3}+(|a|-2|b|+|c|)_{+} / 3<|a|-|b|$ (note this implies $w_{12}>0$ ), then the point $\left(\nu_{3},(|a|-2|b|+|c|)_{+} /\left(3 w_{12}\right)\right)$ does not satisfy the first inequality of (IP: Ib), so $R_{1}$ is a triangle and the optimal value of $\mathcal{P}(\mathbb{R})$ corresponds to the point $\left(\nu_{3},\left(|a|-|b|-w_{23} \nu_{3}\right) / w_{12}\right)$. We can then use Lemma 2.4 to verify that an optimal solution of $\mathcal{I}(\mathbb{Z}, \mathbb{Z})$ is given by $x_{3}=\nu_{3}, x_{6}=$ $\left\lceil\left(|a|-|b|-w_{23} \nu_{3}\right) / w_{12}\right\rceil, y_{1}=0$, and $y_{2}=x_{3}+x_{6}$.

If, on the other hand, $w_{23} \nu_{3}+(|a|-2|b|+|c|)_{+} / 3 \geq|a|-|b|$, then the optimal value of $\mathcal{P}(\mathbb{R})$ corresponds to a point on the lower edge of $R_{1}$, as depicted in Figure (It is worth noting that the point $\left(0,(|a|-2|b|+|c|)_{+} /\left(3 w_{12}\right)\right)$ never satisfies the first inequality of (IP: Ib).) There does not seem to be an exact expression for the minimum coalition size in this case, but we have the following fast method for computing it. The least possible value of $x_{6}$ is $x_{6}^{*}=\left(1+\left\lfloor(|a|-2|b|+|c|) /\left(3 w_{12}\right)\right\rfloor\right)_{+}$(or 0 if $\left.w_{12}=0\right)$. Consider in turn the values $x_{6}=x_{6}^{*}, \ldots, \nu_{6}$. For a given value of $x_{6}$, the least possible value of $x_{3}$ is $x_{3}=\left\lceil\left(|a|-|b|-w_{12} x_{6}\right) / w_{23}\right\rceil$; if this is strictly less than $\left(|b|-|c|+2 w_{12} x_{6}\right) / w_{23}$, then these values of $x_{3}$ and $x_{6}$ along with $y_{1}=0$ and $y_{2}=x_{3}+x_{6}$ are a feasible point of $\mathcal{I}(\mathbb{Z}, \mathbb{Z})$. Moreover, the first feasible point found by this method will be optimal for $\mathcal{I}(\mathbb{Z}, \mathbb{Z})$. This is easy to see. Suppose that another point $\left(x_{3}^{\prime}, x_{6}^{\prime}\right)$ with $x_{6}^{\prime}>x_{6}$ and $x_{3}^{\prime}+x_{6}^{\prime}<x_{3}+x_{6}$ is also the projection of a feasible point for $(\mathcal{I}(\mathbb{Z}, \mathbb{Z}))$. Then

$$
\begin{aligned}
w_{23} x_{3}^{\prime}+w_{12} x_{6}^{\prime} & \leq w_{23} x_{3}+w_{12} x_{6}+w_{23}\left(x_{3}^{\prime}-x_{3}+x_{6}^{\prime}-x_{6}\right) \\
& \leq w_{23} x_{3}+w_{12} x_{6}-w_{23} \\
& <|a|-|b|
\end{aligned}
$$

since $\left(x_{3}-1, x_{6}\right)$ is infeasible for $(\mathcal{P}(\mathbb{R}))$.

In this hard case, $\mathcal{I}(\mathbb{Z}, \mathbb{Z})$ may be infeasible even if $\mathcal{P}(\mathbb{R})$ is feasible (an example is given in Example 2.6); the iterative method of the previous paragraph will then reach $x_{6}=\nu_{6}$ without finding any solution.

The analysis above yields the following result.

Theorem 2.9. If $w_{12} \geq w_{23}$ or $w_{12}=0$, then there is a solution to (IP: Ib) if and only if there is a solution to (PROJ: Ib). Furthermore, the optimal value of (IP: $\mathrm{Ib}$ ) is the greatest integer not exceeding the optimal value of (PROJ: Ib). 
Proof. If $w_{12} \geq w_{23}$, this follows from the easy-case arguments above.

For $w_{12}=0$ (the anti-plurality rule), we must consider the hard case. Without loss of generality, $w_{23}=1$. If $|a|-2|b|+|c| \geq 0$ or $\nu_{3}<|a|-|b|, \mathcal{P}(\mathbb{R})$ is infeasible. Otherwise, $\mathcal{P}(\mathbb{R})$ is feasible, and we must apply the iterative algorithm discussed above. We begin with $x_{6}=x_{6}^{*}=0$ and corresponding $x_{3}=|a|-|b|$; since this is less than $|b|-|c|$, we have immediately found an optimal solution of $\mathcal{I}(\mathbb{Z}, \mathbb{Z})$. The optimal value for both $\mathcal{I}(\mathbb{Z}, \mathbb{Z})$ and $\mathcal{P}(\mathbb{R})$ is $|a|-|b|$.

\section{Description of the algorithm}

Our exact results are of course based on enumeration of voting situations. However, our tie-breaking methods allow us to make use of symmetry to reduce the search space, as we now describe.

The group $G$ of all permutations of the candidates acts on the set $\mathcal{S}$ of voting situations in a natural way. Whether a voting situation is manipulable is invariant under permutations of the candidates, so is a property of an orbit of $G$ on $\mathcal{S}$.

Clearly, we would like to examine only one representative from each $G$-orbit on $\mathcal{S}$. However, a simple rule for carrying this out is not apparent. Below we give a simple rule that in most cases chooses a single representative, but in the worst case may choose the entire orbit.

Theorem 3.1. Let $F=\{\sigma \in \mathcal{S}:|a| \geq|b| \geq|c|\}$. Define $c: \mathcal{S} \rightarrow \mathcal{Z}$ by

$$
c(\sigma)= \begin{cases}\frac{m !}{\prod_{i} l_{i} !} & \text { where the } l_{i} \text { are the multiplicities of the distinct scores, if } \sigma \in F \\ 0 & \text { otherwise. }\end{cases}
$$

Then for each $G$-invariant function $\mu$ on $\mathcal{S}$, we have

$$
\sum_{\sigma \in \mathcal{S}} c(x) \mu(x)=\sum_{\sigma \in \mathcal{S}} \mu(x)
$$

Proof. The group $G$ acts naturally on the scoreboards, and the score map taking voting situations to scoreboards commutes with the action of $G$. Thus each $G$-orbit $\mathcal{O}$ on $\mathcal{S}$ is mapped onto a $G$-orbit of scoreboards.

Now $\mathcal{O}$ contains at least one element $x$ such that its scoreboard $s$ is in nonincreasing order, $|a| \geq$ $|b| \geq|c|$. The size of the $G$-orbit of $s$ is precisely $c(x)$. Also $\mathcal{O}$ is the disjoint union of fibres $\alpha^{-1}(t)$ of the score map, and there are $c(x)$ of these. Furthermore all fibres have the same cardinality. Thus we have

$$
\sum_{z \in \mathcal{O}} c(z) \mu(z)=\mu(x) \sum_{z \in \mathcal{O}} c(z)=\mu(x) \sum_{z \in \mathcal{O}, \alpha(z)=s} c(z)=\mu(x) c(x)\left|\alpha^{-1}(s)\right|=\mu(x)|\mathcal{O}|=\sum_{z \in \mathcal{O}} \mu(z),
$$

and summing over all $G$-orbits gives the result.

Example 3.2. Consider an election under the Borda rule. If the voting situation is $(1,0,0,1,1,0)$, its $G$-orbit has size 2 and each element has scoreboard $(3,3,3)$, so has $c=1$. If the voting situation is $(0,1,2,0,0,0)$, then its $G$-orbit has size 6 . There are two elements, namely $(0,1,2,0,0,0)$ and $(2,0,0,0,1,0)$, that have scoreboard $(4,4,1)$, and each has $c=3$. The voting situation $(2,1,0,0,0,0)$ has $G$-orbit of size 6 and scoreboard $(6,2,1)$, and receives the value $c=6$.

We shall apply Theorem 3.1 with $\mu$ equal to the indicator function of manipulability (when counting manipulable situations), and also with $\mu$ equal to the minimum size of a manipulating coalition. 
Note that we need only consider voting situations whose scoreboard satisfies $|a| \geq|b| \geq|c|$. Thus our algorithm makes use of the theorem to cut the number of voting situations that must be examined, by a factor slightly less than 6 . Voting situations are generated systematically. For each situation, we compute the scoreboard and the value of $c$ above. If the scoreboard is not decreasing $(c=0)$, we move to the next voting situation. If $c \neq 0$, we compute the minimum coalition size, or report that manipulation is infeasible. This computation follows the description in Section 2.2. We consider type II or type I manipulations depending on whether $|a|=|b|$ or not. We need to compute only the standard manipulations (for type I, this is manipulation making $b$ a winner), and apply the appropriate permutation to reduce others to this case.

We have implemented the algorithm in $\mathrm{C}++$ (code is available from the authors on request).

\section{Selected numerical results}

The algorithm described in Section 3 allows us to compute, for each voting situation, whether the situation can be manipulated by a coalition of voters, and the minimum size of such a manipulating coalition.

To gain an overview of manipulability, we must make some assumption as to which voting situations are the most likely to occur. We will use two of the most common models for this:

- IAC (Impartial Anonymous Culture): all voting situations are equally likely.

- IC (Impartial Culture): all profiles are equally likely. Note that a voting situation $\left(\nu_{1}, \ldots, \nu_{M}\right)$ (with a total of $n$ voters) represents $n ! /\left(\nu_{1} ! \cdots \nu_{M} !\right)$ profiles.

The IC model can be described probabilistically: voters act independently, and each voter is equally likely to choose any of the possible preference orders. In all but the smallest electorates, this means (due to the law of large numbers) that most of the probability is placed on voting situations where all candidates have roughly equal support, and the margin of victory is small. Such situations are often the most prone to manipulation. In contrast, the IAC model places more probability on voting situations where the margin of victory is relatively large, and manipulation may be more difficult.

With our culture model (IAC or IC) and a voting rule chosen, let $f_{n}(k)$ denote the probability that an election involving $n$ voters and 3 candidates is manipulable by a coalition of $k$ or fewer voters. Previous work on manipulation has focused mainly on the extremes $k=1$ (manipulation by individuals) and $k=n$ (manipulation by any coalition); we will consider all values of $k$. Thus we compute the full probability distribution function $f_{n}$ of the random variable $M$, which equals the minimal coalition size (or $\infty$ if manipulation is not possible). We restrict to $k \leq n / 2$ because in every example we have seen, increasing $k$ does not lead to any change in the probability (we do not have a formal proof of the plausible statement that the minimum size of a manipulating coalition is always at most $n / 2$ ).

Tables 2 and 3 give the values of $f_{n}(k)$ for small numbers of voters, for several positional voting rules. Tables 4 and 5 consider manipulability in a large $(n=150)$ population of voters.

These tables of $f_{n}(k)$ for small $n$ show that the likelihood of manipulation in small committees is high. Furthermore we see that some rules are completely dominated by others (in the sense that for each $k$ and $\left.n, f_{n}(k) \leq g_{n}(k)\right)$.

It turns out that each $n \leq 12$, there is often a single dominant rule among the six rules displayed here. These are shown in Table 6 and Table 7. We also include extra information in these tables for discussion in the next section.

Note that under IAC, the rule specified by $(3,2,0)$ is completely dominated by Borda, plurality and the $(3,1,0)$ rule over the entire range $n \leq 12$. Plurality also dominates the $(10,9,0)$ and antiplurality 
Table 2: Manipulability of various rules for small numbers of voters, under IAC behaviour.

\begin{tabular}{|c|c|c|c|c|c|c|c|}
\hline$n$ & $k$ & plurality & $(3,1,0)$ & Borda & $(3,2,0)$ & $(10,9,0)$ & anti-plurality \\
\hline 2 & 1 & 0.000000 & 0.428571 & 0.142857 & 0.142857 & 0.142857 & 0.428571 \\
\hline 3 & 1 & 0.000000 & 0.000000 & 0.321429 & 0.321429 & 0.321429 & 0.214286 \\
\hline \multirow[t]{2}{*}{4} & 1 & 0.214286 & 0.333333 & 0.190476 & 0.357143 & 0.357143 & 0.404762 \\
\hline & 2 & 0.214286 & 0.428571 & 0.190476 & 0.357143 & 0.357143 & 0.404762 \\
\hline \multirow[t]{2}{*}{5} & 1 & 0.214286 & 0.238095 & 0.309524 & 0.333333 & 0.261905 & 0.452381 \\
\hline & 2 & 0.214286 & 0.333333 & 0.380952 & 0.404762 & 0.285714 & 0.452381 \\
\hline \multirow[t]{3}{*}{6} & 1 & 0.155844 & 0.227273 & 0.233766 & 0.324675 & 0.305195 & 0.305195 \\
\hline & 2 & 0.207792 & 0.305195 & 0.350649 & 0.441558 & 0.474026 & 0.305195 \\
\hline & 3 & 0.207792 & 0.344156 & 0.350649 & 0.441558 & 0.474026 & 0.305195 \\
\hline \multirow[t]{3}{*}{7} & 1 & 0.242424 & 0.219697 & 0.250000 & 0.280303 & 0.303030 & 0.393939 \\
\hline & 2 & 0.242424 & 0.310606 & 0.340909 & 0.393939 & 0.424242 & 0.484848 \\
\hline & 3 & 0.242424 & 0.363636 & 0.363636 & 0.401515 & 0.431818 & 0.484848 \\
\hline \multirow[t]{4}{*}{8} & 1 & 0.205128 & 0.202797 & 0.223776 & 0.282051 & 0.247086 & 0.396270 \\
\hline & 2 & 0.263403 & 0.291375 & 0.340326 & 0.421911 & 0.340326 & 0.466200 \\
\hline & 3 & 0.286713 & 0.347319 & 0.386946 & 0.473193 & 0.349650 & 0.466200 \\
\hline & 4 & 0.286713 & 0.361305 & 0.386946 & 0.473193 & 0.349650 & 0.466200 \\
\hline \multirow[t]{4}{*}{9} & 1 & 0.194805 & 0.182817 & 0.224775 & 0.236763 & 0.260739 & 0.299700 \\
\hline & 2 & 0.224775 & 0.272727 & 0.332667 & 0.359640 & 0.419580 & 0.353646 \\
\hline & 3 & 0.224775 & 0.323676 & 0.404595 & 0.410589 & 0.488511 & 0.353646 \\
\hline & 4 & 0.224775 & 0.350649 & 0.416583 & 0.422577 & 0.488511 & 0.353646 \\
\hline \multirow[t]{5}{*}{10} & 1 & 0.209790 & 0.185814 & 0.196803 & 0.248751 & 0.251748 & 0.349650 \\
\hline & 2 & 0.257742 & 0.273726 & 0.304695 & 0.387612 & 0.395604 & 0.471528 \\
\hline & 3 & 0.281718 & 0.336663 & 0.362637 & 0.463536 & 0.457542 & 0.511489 \\
\hline & 4 & 0.293706 & 0.374625 & 0.388611 & 0.481518 & 0.461538 & 0.511489 \\
\hline & 5 & 0.293706 & 0.382617 & 0.388611 & 0.481518 & 0.461538 & 0.511489 \\
\hline \multirow[t]{5}{*}{11} & 1 & 0.200549 & 0.170330 & 0.200549 & 0.225275 & 0.221154 & 0.343407 \\
\hline & 2 & 0.250000 & 0.258242 & 0.302198 & 0.353022 & 0.335165 & 0.442308 \\
\hline & 3 & 0.266484 & 0.318681 & 0.384615 & 0.432692 & 0.380495 & 0.475275 \\
\hline & 4 & 0.266484 & 0.357143 & 0.427198 & 0.457418 & 0.385989 & 0.475275 \\
\hline & 5 & 0.266484 & 0.370879 & 0.434066 & 0.462912 & 0.385989 & 0.475275 \\
\hline \multirow[t]{6}{*}{12} & 1 & 0.180349 & 0.160957 & 0.180349 & 0.209438 & 0.223497 & 0.276341 \\
\hline & 2 & 0.231739 & 0.247253 & 0.286037 & 0.336458 & 0.372818 & 0.356820 \\
\hline & 3 & 0.255495 & 0.307369 & 0.361183 & 0.421299 & 0.462993 & 0.383969 \\
\hline & 4 & 0.269069 & 0.351002 & 0.414512 & 0.463963 & 0.501778 & 0.383969 \\
\hline & 5 & 0.275856 & 0.374273 & 0.429056 & 0.472689 & 0.501778 & 0.383969 \\
\hline & 6 & 0.275856 & 0.379121 & 0.429056 & 0.472689 & 0.501778 & 0.383969 \\
\hline
\end{tabular}


Table 3: Manipulability of various rules for small numbers of voters, under IC behaviour.

\begin{tabular}{|c|c|c|c|c|c|c|c|}
\hline$n$ & $k$ & plurality & $(3,1,0)$ & Borda & $(3,2,0)$ & $(10,9,0)$ & anti-plurality \\
\hline 2 & 1 & 0.000000 & 0.500000 & 0.166667 & 0.166667 & 0.166667 & 0.333333 \\
\hline 3 & 1 & 0.000000 & 0.000000 & 0.250000 & 0.250000 & 0.250000 & 0.111111 \\
\hline \multirow[t]{2}{*}{4} & 1 & 0.333333 & 0.472222 & 0.291667 & 0.402778 & 0.402778 & 0.296296 \\
\hline & 2 & 0.333333 & 0.555556 & 0.291667 & 0.402778 & 0.402778 & 0.296296 \\
\hline \multirow[t]{2}{*}{5} & 1 & 0.370370 & 0.339506 & 0.324074 & 0.408951 & 0.258488 & 0.375000 \\
\hline & 2 & 0.370370 & 0.416667 & 0.416667 & 0.462963 & 0.281636 & 0.375000 \\
\hline \multirow[t]{3}{*}{6} & 1 & 0.246914 & 0.353652 & 0.331147 & 0.362011 & 0.329604 & 0.175412 \\
\hline & 2 & 0.308642 & 0.453961 & 0.423740 & 0.516332 & 0.507073 & 0.175412 \\
\hline & 3 & 0.308642 & 0.471965 & 0.423740 & 0.516332 & 0.507073 & 0.175412 \\
\hline \multirow[t]{3}{*}{7} & 1 & 0.462106 & 0.357082 & 0.336077 & 0.380187 & 0.384538 & 0.347951 \\
\hline & 2 & 0.462106 & 0.477109 & 0.471858 & 0.514168 & 0.510867 & 0.394762 \\
\hline & 3 & 0.462106 & 0.537873 & 0.503365 & 0.518669 & 0.515368 & 0.394762 \\
\hline \multirow[t]{4}{*}{8} & 1 & 0.384088 & 0.348330 & 0.335002 & 0.368809 & 0.273773 & 0.374200 \\
\hline & 2 & 0.468107 & 0.466357 & 0.478134 & 0.546550 & 0.335787 & 0.397005 \\
\hline & 3 & 0.484111 & 0.522369 & 0.525145 & 0.610565 & 0.341789 & 0.397005 \\
\hline & 4 & 0.484111 & 0.525120 & 0.525145 & 0.610565 & 0.341789 & 0.397005 \\
\hline \multirow[t]{4}{*}{9} & 1 & 0.384088 & 0.327300 & 0.330050 & 0.327725 & 0.322297 & 0.204703 \\
\hline & 2 & 0.432099 & 0.468557 & 0.479260 & 0.498189 & 0.502649 & 0.214991 \\
\hline & 3 & 0.432099 & 0.549576 & 0.545525 & 0.582808 & 0.571465 & 0.214991 \\
\hline & 4 & 0.432099 & 0.578457 & 0.556777 & 0.594061 & 0.571465 & 0.214991 \\
\hline \multirow[t]{5}{*}{10} & 1 & 0.434099 & 0.335768 & 0.324503 & 0.348929 & 0.341792 & 0.354003 \\
\hline & 2 & 0.498114 & 0.469403 & 0.479018 & 0.533352 & 0.505874 & 0.434432 \\
\hline & 3 & 0.522119 & 0.547713 & 0.570539 & 0.613724 & 0.561002 & 0.443863 \\
\hline & 4 & 0.526120 & 0.572677 & 0.597545 & 0.624477 & 0.562669 & 0.443863 \\
\hline & 5 & 0.526120 & 0.573327 & 0.597545 & 0.624477 & 0.562669 & 0.443863 \\
\hline \multirow[t]{5}{*}{11} & 1 & 0.418585 & 0.328479 & 0.317480 & 0.331406 & 0.268390 & 0.362999 \\
\hline & 2 & 0.535945 & 0.481467 & 0.471247 & 0.528225 & 0.357605 & 0.406740 \\
\hline & 3 & 0.554282 & 0.566568 & 0.574720 & 0.634460 & 0.375834 & 0.411521 \\
\hline & 4 & 0.554282 & 0.613192 & 0.613168 & 0.673160 & 0.377323 & 0.411521 \\
\hline & 5 & 0.554282 & 0.624003 & 0.616720 & 0.676140 & 0.377323 & 0.411521 \\
\hline \multirow[t]{6}{*}{12} & 1 & 0.399025 & 0.307993 & 0.310184 & 0.300434 & 0.299059 & 0.218128 \\
\hline & 2 & 0.465040 & 0.461212 & 0.464178 & 0.472296 & 0.478058 & 0.240433 \\
\hline & 3 & 0.489490 & 0.551249 & 0.579748 & 0.589853 & 0.575725 & 0.242694 \\
\hline & 4 & 0.496825 & 0.598379 & 0.632886 & 0.640546 & 0.603008 & 0.242694 \\
\hline & 5 & 0.497803 & 0.614579 & 0.644148 & 0.650861 & 0.603008 & 0.242694 \\
\hline & 6 & 0.497803 & 0.614724 & 0.644148 & 0.650861 & 0.603008 & 0.242694 \\
\hline
\end{tabular}

Table 4: Manipulability of various rules for a large number (150) of voters, under IAC behaviour.

\begin{tabular}{|c|c||c|c|c|c|c|c|}
\hline$n$ & $k$ & plurality & $(3,1,0)$ & Borda & $(3,2,0)$ & $(10,9,0)$ & anti-plurality \\
\hline 150 & 1 & 0.028768 & 0.019740 & 0.020304 & 0.024294 & 0.027861 & 0.041790 \\
& 10 & 0.156653 & 0.141459 & 0.137456 & 0.184919 & 0.232361 & 0.248109 \\
& 20 & 0.231809 & 0.240621 & 0.253923 & 0.329686 & 0.390713 & 0.395509 \\
& 50 & 0.289056 & 0.402909 & 0.474906 & 0.531345 & 0.536958 & 0.504149 \\
& $\infty$ & 0.291722 & 0.425031 & 0.496558 & 0.537954 & 0.537175 & 0.504149 \\
\hline
\end{tabular}


Table 5: Manipulability of various rules for a large number (150) of voters, under IC behaviour.

\begin{tabular}{|c|c||c|c|c|c|c|c|}
\hline$n$ & $k$ & plurality & $(3,1,0)$ & Borda & $(3,2,0)$ & $(10,9,0)$ & anti-plurality \\
\hline 150 & 1 & 0.167757 & 0.108331 & 0.100657 & 0.105540 & 0.108635 & 0.140902 \\
& 10 & 0.819020 & 0.695129 & 0.590518 & 0.641616 & 0.570225 & 0.407870 \\
& 20 & 0.975120 & 0.937504 & 0.874442 & 0.894358 & 0.729099 & 0.413466 \\
& 50 & 0.981641 & 0.994959 & 0.996119 & 0.994388 & 0.830657 & 0.413468 \\
& $\infty$ & 0.981641 & 0.994975 & 0.996128 & 0.994389 & 0.830657 & 0.413468 \\
\hline
\end{tabular}

Table 6: Best rules for $n \leq 12$, by various measures, under IAC

\begin{tabular}{|c|c|c|c|c|c|c|c|c|c|c|c|}
\hline Number of voters & 2 & 3 & 4 & 5 & 6 & 7 & 8 & 9 & 10 & 11 & 12 \\
\hline Dominant rule & plur & plur & Borda & plur & plur & none & none & none & none & none & none \\
\hline $\operatorname{Pr}(M=1)$ least & plur & plur & Borda & plur & plur & $(3,1,0)$ & $(3,1,0)$ & $(3,1,0)$ & $(3,1,0)$ & $(3,1,0)$ & $(3,1,0)$ \\
\hline $\operatorname{Pr}(M<\infty)$ least & plur & plur & Borda & plur & plur & plur & plur & plur & plur & plur & plur \\
\hline
\end{tabular}

over this range. It seems that under IAC, the "easy" case rules are much superior to the "hard" ones. The reverse is true (to a less extent) under IC however: for $6 \leq n \leq 12$, plurality is dominated by antiplurality. However, note that again the $(3,2,0)$ rule is uncompetitive, being dominated by Borda for $n \leq 11$, and by at least one other "easy case" rule for each $n \leq 12$.

We now move on to larger values of $n$. In Figure 4 we plot $\operatorname{Pr}(M<\infty)$ for $n \leq 50$. In Figure 4, we graph $f_{n}(k)$ against $k$ for $n=48, n=49, n=50$. The mod-3 periodicity evident for smaller $n$ is still very clear for the hard case rules but not in the easy case. Integer effects are more important in the hard case, as we saw in Section 2.3.

\section{Comparison with the existing literature}

The present article is the first to provide exact (computational) results for positional rules other than the standard three (plurality, Borda, antiplurality). Very few authors have considered quantitative measures of manipulability for a general positional rule. Furthermore our random tiebreaking has not been commonly used. Thus, though we believe our approach to be superior to those undertaken by previous authors, direct comparison with existing results is not easy. Below we discuss a few cases in which such comparison can be profitably made. We discuss both results and methodology.

Previous authors have usually considered one or more rules, which need not be positional; the positional rules chosen have been limited to plurality, Borda, and antiplurality. Furthermore, they

- use various definitions of manipulability;

- consider various measures of manipulability;

- give statistical results for these measures that are either

Table 7: Best rules for $n \leq 12$, by various measures, under IC

\begin{tabular}{|c|c|c|c|c|c|c|c|c|c|c|c|}
\hline Number of voters & 2 & 3 & 4 & 5 & 6 & 7 & 8 & 9 & 10 & 11 & 12 \\
\hline Dominant rule & plur & plur & Borda & $(10,9,0)$ & antip & none & $(10,9,0)$ & antip & none & $(10,9,0)$ & antip \\
\hline $\operatorname{Pr}(M=1)$ least & plur & plur & Borda & $(10,9,0)$ & antip & Borda & $(10,9,0)$ & antip & Borda & $(10,9,0)$ & antip \\
\hline $\operatorname{Pr}(M<\infty)$ least & plur & plur & Borda & $(10,9,0)$ & antip & antip & $(10,9,0)$ & antip & antip & $(10,9,0)$ & antip \\
\hline
\end{tabular}




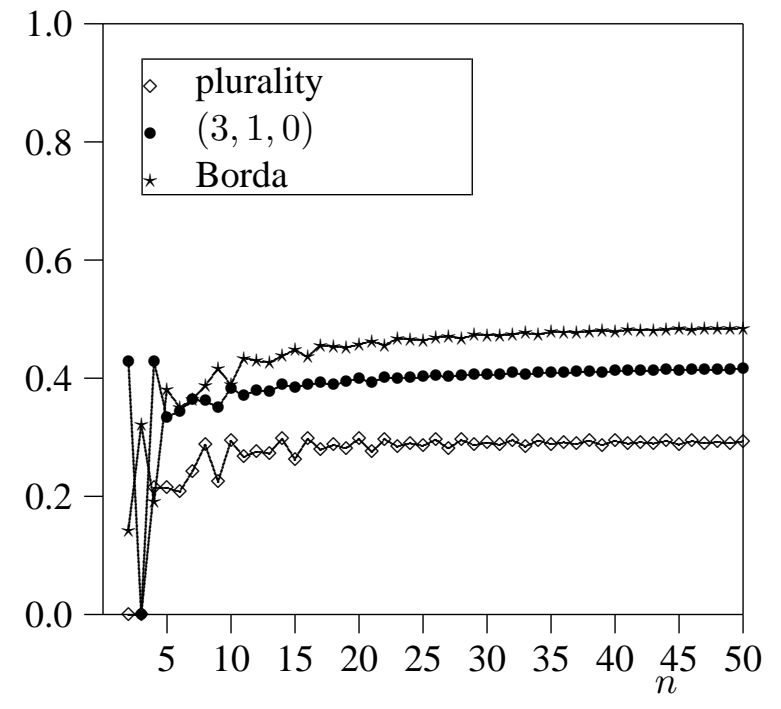

"Easy" rules, IAC behaviour

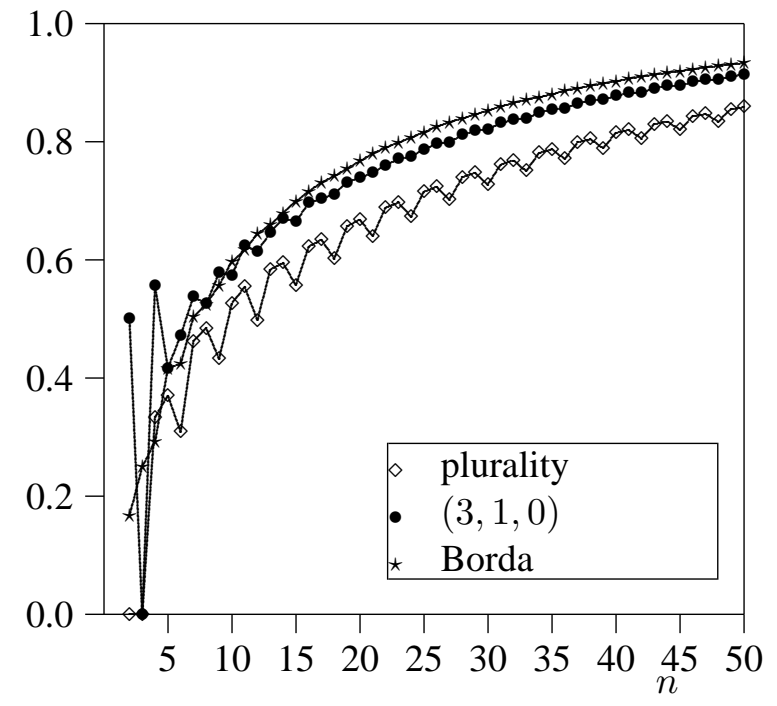

"Easy" rules, IC behaviour

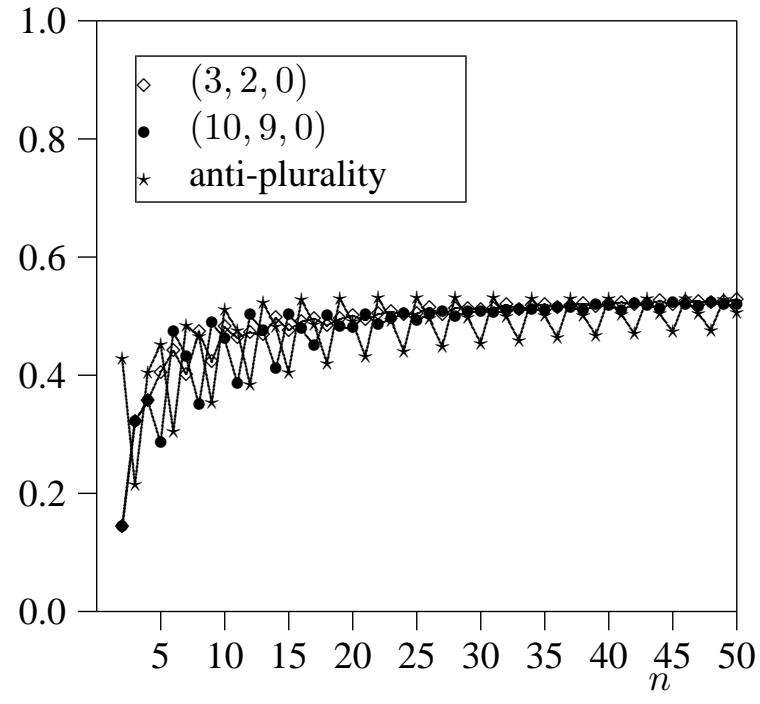

"Hard" rules, IAC behaviour

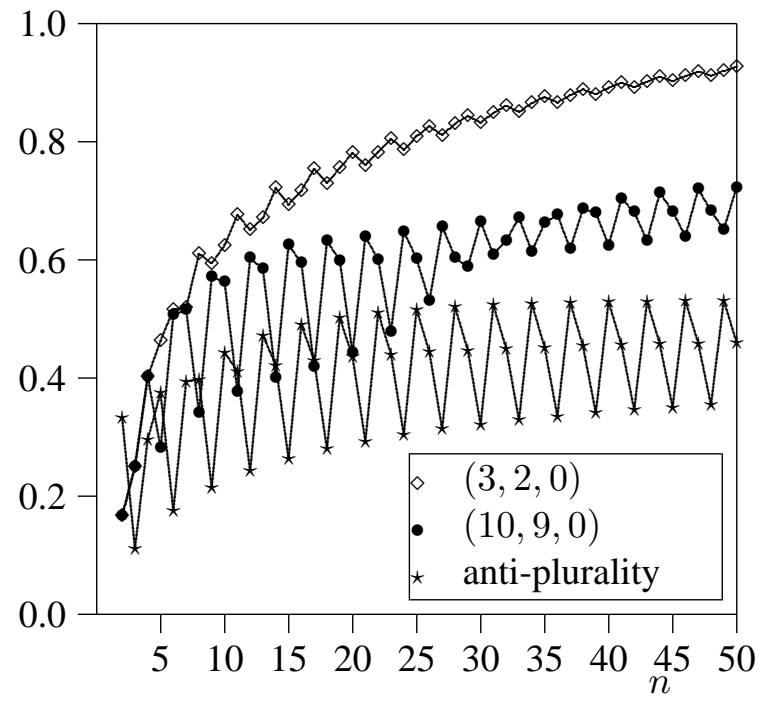

"Hard" rules, IC behaviour

Figure 3: Probability of the occurrence of a manipulable situation, as a function of the number of voters 


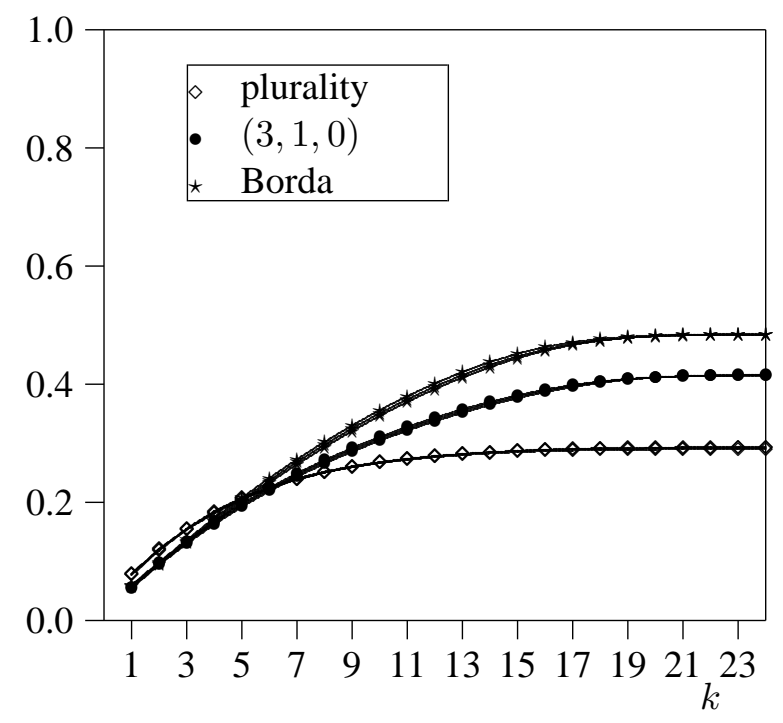

"Easy" rules, IAC behaviour

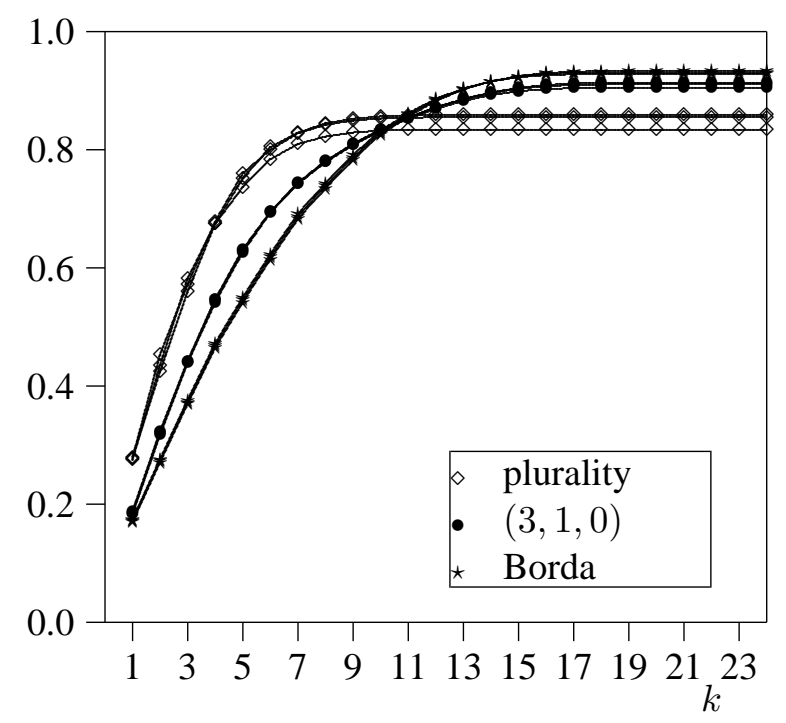

"Easy" rules, IC behaviour

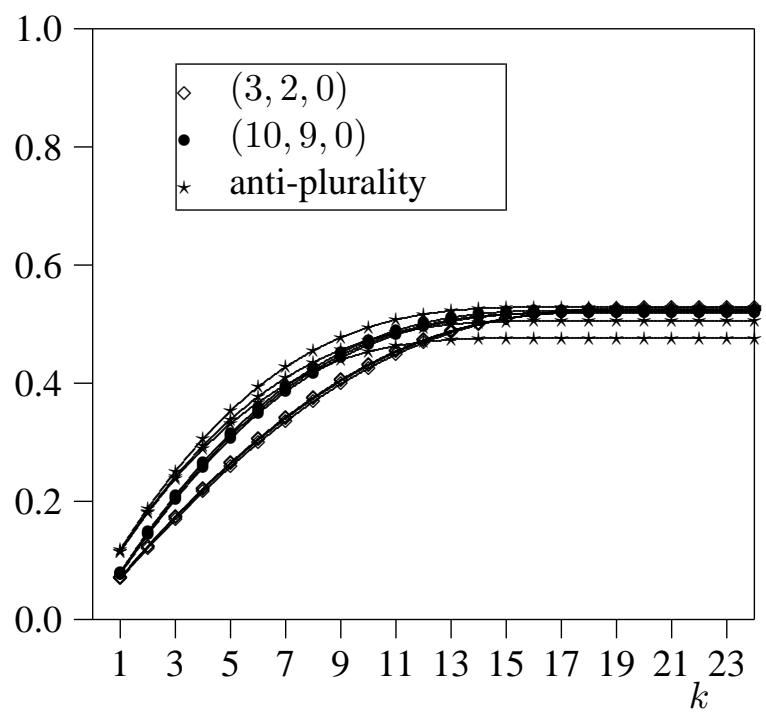

"Hard" rules, IAC behaviour

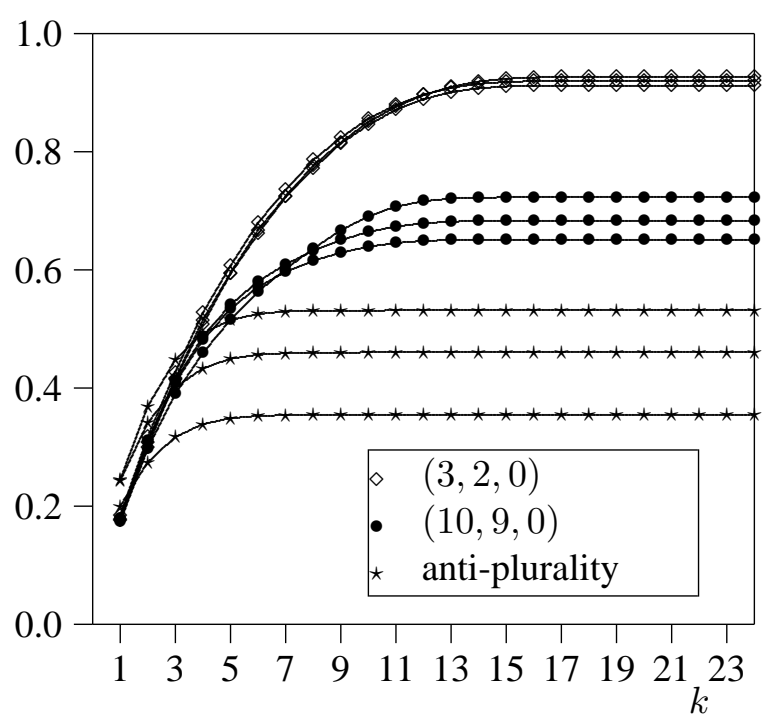

"Hard" rules, IC behaviour

Figure 4: Manipulability by coalitions of various sizes, for 48,49 , or 50 voters 
- asymptotic (as the number of voters tends to $\infty$ );

- exact analytic (given by a formula for finite $n$ );

- exact computational (derived by an algorithm, as in the present article);

- based on random sampling;

- consider various probability distributions on voter preferences (of which the Impartial Culture and Impartial Anonymous Culture models are the most common by far).

We discuss each of these issues except the last in more detail below. Results for the three standard positional rules (and other non-positional rules) for more general preference models are presented in [11]. In [2] a so-called spatial model of voting is used. Apart from these, to our knowledge all papers have dealt with IC or IAC only, and we confine our discussion to these cases.

\subsection{Definitions of manipulability}

Various definitions of manipulability are used in the literature (and we discuss them below). Small differences in such definitions, and issues such as tiebreaking, will not affect our results substantially when the number $n$ of voters is large, although tiebreaking does affect numerical results for small $n$. Most previous authors have used lexicographical tiebreaking.

There are two main distinctions to make. First, there is individual manipulation (in other words, by a coalition of size 1) versus more general coalitional manipulation. Individual manipulation only was studied in earlier papers while recently coalitional manipulation has been more studied. Second, there is a big difference between naive manipulation (when the manipulating voters ignore any possible gameplaying by other voters) and the more sophisticated type of manipulation that allows for the possibility of reversals or counterthreats. The only quantitative works on manipulability in the latter case of which we are aware are [10,3], which also consider naive manipulation; all others (including ourselves) use only the naive concept.

Some authors (for example Saari [14]) have studied a different concept under the name of manipulation, which we do not consider to be a valid manipulation in general. Namely, one can consider the easier problem of promoting $b$ ahead of $a$, without worrying that $c$ may thereby overtake $b$. This is called threshold manipulability in [13] and is simpler to analyse.

Technically, our random tiebreaking does not specify a voting rule. A rule is called resolute if it always produces a unique winner from each voting situation. Ties can be handled by considering nonresolute rules. In [20] several different definitions of manipulation of non-resolute rules are presented. It is not at all clear to us which, if any, of these definitions coincides with our definition.

\subsection{Measures of manipulability}

Several measures or indices of manipulability are used in [7, 1, 19, 2], all of which deal only with IC and use random sampling (the first three cited articles deal only with individual manipulation). These include such measures as the number of candidates in whose favour manipulation is possible, the "margin of error" of an attempted manipulation, and the number of voters who can individually manipulate. All these measures can be computed in a straightforward way by modifying our program; however we have not implemented these additional capabilities.

To our knowledge all other papers fit into the following framework. Let $M$ be the minimal size of a manipulating coalition. Early papers considered the probability $\operatorname{Pr}(M=1)$; that is, they considered only manipulability by a single voter. Later papers have considered the logical possibility of coalitional 
Table 8: Probabilities of manipulability under IAC

\begin{tabular}{|c|c|c|c|c|}
\hline Rule & $\lim _{n} \operatorname{Pr}(M<\infty)$ & exact formula? & $\operatorname{Pr}(M=1)$ & exact formula? \\
\hline Positional & $0 \leq c \leq 1$ & no & $\mathcal{O}\left(n^{-1}\right)[18]$ & no \\
\hline Plurality & $7 / 24 \approx 0.2917[8]$ & yes [8] & $\sim(55 / 18) n^{-1}$ & yes [10] \\
\hline Borda & $\approx 0.5025[4]$ & no & $\sim(25 / 12) n^{-1}$ & yes [4] \\
\hline Antiplurality & $14 / 27 \approx 0.5185$ & yes [6] & $?$ & $?$ \\
\hline
\end{tabular}

Table 9: Probabilities of manipulability under IC

\begin{tabular}{|c|c|c|c|c|}
\hline Rule & $\operatorname{Pr}(M<\infty)$ & exact formula? & $\operatorname{Pr}(M=1)$ & exact formula? \\
\hline Positional & $\rightarrow 1$ exp. fast & no & $\mathcal{O}\left(n^{-1 / 2}\right)[17]$ & no \\
\hline Plurality & $\rightarrow 1$ & $?$ & $?$ & $?$ \\
\hline Borda & $\rightarrow 1$ & $?$ & $?$ & $?$ \\
\hline Antiplurality & $\rightarrow 1 / 2$ & $?$ & $?$ & $?$ \\
\hline
\end{tabular}

manipulation; that is, $\operatorname{Pr}(M<\infty)$. In [2] the average minimum coalition size (conditional on manipulation being possible, that is, $E[M \mid M<\infty]$ ) was studied. The average threshold coalition size was discussed in [13].

Our analysis in the present article yields the full probability distribution of the minimum coalition size, since we can compute $\operatorname{Pr}(M \leq k)$ for each $k$. Thus we can readily compute any of the above statistics if desired.

\subsection{Statistical results}

We summarize some known results in Table 8 and Table 9. A question mark "?" means that we do not know whether the relevant entry is known; by "exact formula" we mean a formula for finite $n$.

Exact results are found in a few papers. Results obtained by random sampling under IC are presented in $[12,19,1]$. The latter two articles cover Borda and plurality. Several papers $[8,10,9,11,4,3]$ present exact analytic and computational results for IAC, for the three standard positional rules.

We first consider $\operatorname{Pr}(M=1)$. The results of the above-mentioned papers sometimes vary substantially from ours. In [19], for Borda and $n=11$ the value 0.2321 is given, whereas we obtain 0.3175 ; for $n=50$ the corresponding values are 0.1159 and 0.1701 . However, it is easily seen that the probability of manipulability under our random tiebreaking assumption will always exceed that computed under the assumption of lexicographic tiebreaking, so these results are consistent.

Under IC, for every positional rule except antiplurality, $\operatorname{Pr}(M<\infty)$ approaches 1 exponentially fast as $n \rightarrow \infty$, while it approaches $1 / 2$ in the case of antiplurality. This convergence is certainly consistent with Table 5. When $n=21$, the paper [2] gives $\operatorname{Pr}(M<\infty)$ as 0.810 for plurality and 0.960 for Borda, whereas we obtain 0.640 and 0.780 respectively. We do not have an explanation for this discrepancy; it is certainly true that our tiebreaking assumptions are not exactly the same.

By contrast, under IAC $\operatorname{Pr}(M<\infty)$ converges to a constant between 0 and 1 . These constants do not depend on the tie-breaking rules, since the set of tied situations has asymptotic negligible probability. Our results agree well with these limits for $\operatorname{Pr}(M<\infty)$. For example, when $n=150$ we have $0.2917,0.4966,0.5041$ for plurality, Borda, antiplurality respectively. Note that convergence for the latter is slower, as may be expected.

The other statistic that has been used is $E[M \mid M<\infty]$, the expected minimum size of a manipulating coalition. This was introduced in [2]. The numerical results presented there are not easily verified, because a slightly different measure is computed. See Section 6 for more discussion of this measure. 


\section{Extensions and future work}

There are many areas for further study, and we now list some.

- One obvious area is to understand better the type III manipulations. Here we derive the appropriate linear system and record it for possible future use. Consider Case 2(iv). The coalition members must prefer $c$ to $b$, so we have $x_{1}=x_{3}=x_{4}=0$. Let $y_{1}, y_{2}, y_{3}, y_{4}, y_{5}$, and $y_{6}$ denote the numbers of coalition members who strategically vote $a b c, a c b, b a c, b c a, c a b$, and $c b a$ respectively. The conditions to be satisfied are then derived as above:

$$
\begin{aligned}
& 0=|b|-|c|+w_{21} x_{2}+w_{12} x_{5}+w_{13} x_{6}+w_{13} y_{1}+w_{12} y_{2}+w_{23} y_{3}+w_{32} y_{4}+w_{21} y_{5}+w_{31} y_{6} \\
& \text { (IP:IIIc) } \\
& 0<|c|-|b|+w_{32} x_{2}+w_{31} x_{5}+w_{21} x_{6}+w_{32} y_{1}+w_{23} y_{2}+w_{31} y_{3}+w_{21} y_{4}+w_{13} y_{5}+w_{12} y_{6} \\
& 0=x_{2}+x_{5}+x_{6}-y_{1}-y_{2}-y_{3}-y_{4}-y_{5}-y_{6} \\
& 0 \leq x_{i} \leq \nu_{i} \text { for } 1 \leq i \leq 6 \\
& 0 \leq y_{j} \text { for } 1 \leq j \leq 6 \\
& \text { all } x_{i}, y_{j} \text { are integers. }
\end{aligned}
$$

Case 2(v) can be obtained from 2(iv) by transposing $a$ and $b$.

- The measure $\operatorname{Pr}(M<\infty)$ can of course be computed for a given $n$ by our methods, since it is just the maximum value of $f_{n}(k)$. If a more analytic result is required, we can easily derive one by specializing our linear systems. Explicitly, for each case listed in Table 1, there is a maximal coalition consisting of all voters with incentive to participate in a coalition. Since some of these can still vote sincerely if they so desire, it follows that a situation is manipulable if and only if it is manipulable by the appropriate maximal coalition. Algebraically, this means that the $x$ 's in systems such as (IP: Ib) are replaced by the corresponding $\nu$ 's. We can also express the scores in terms of the $\nu$ 's by (1). This yields integer linear conditions in the $\nu$ 's and $y$ 's only, which are necessary and sufficient for manipulation to be achievable. The problem now reduces to one of counting lattice points inside a convex region, to which standard techniques can be applied.

Some such conditions have been derived in various special cases by previous authors. One could attempt to perform such a computation for a general positional rule.

- The results for small $n$ immediately suggest conjectures about asymptotic behaviour of rules. For example,

- plurality asymptotically minimizes $\operatorname{Pr}(M<\infty)$ for IAC;

- some easy case rule other than plurality or Borda minimizes $\operatorname{Pr}(M=1)$ asymptotically under IAC;

- Borda is the rule for which $\operatorname{Pr}(M<\infty)$ converges fastest to 1 under IC.

- The measures $\operatorname{Pr}(M=1)$ and $\operatorname{Pr}(M<\infty)$ are the most commonly used in the literature. The measure $E[M \mid M<\infty]$ was introduced in [2]. In [13] several results were derived for a general positional rule under IC. It was shown that this measure is asymptotically equal to $C(p) n^{1 / 2}$ where $C$ depends on $p:=\left(w_{1}-w_{2}\right) /\left(w_{1}-w_{3}\right)$. Furthermore, for easy case rules $(p \geq 1 / 2), C$ behaves continuously in $p$. But for the hard case, even though $C(0)$ is a finite constant, there is a discontinuity of $C$ at $p=0$ : $\lim _{p \rightarrow 0} C(p)=\infty$. Thus the closer a rule approaches antiplurality, the larger is $E[M \mid M<\infty]$. Hence this measure is probably not all that useful, assuming IC. However, under IAC we expect the measure to be of order $n$, and it may be of more use. 
- We note that for many non-positional voting rules we can use the same methodology of deriving linear systems to describe manipulability. Similarly, the type of linear systems derived here also occur in other areas of voting theory.

\section{References}

[1] Fuad T. Aleskerov and Eldaniz Kurbanov. A degree of manipulability of known social choice procedures. In Ahmet Alkan, Charalambos Aliprantis, and Nicholas Yannelis, editors, Current Trends in Economics: Theory and Applications, pages 13-27. Springer, 1999.

[2] John R. Chamberlin. An investigation into the relative manipulability of four voting systems. Behavioral Sci., 30(4):195-203, 1985.

[3] Pierre Favardin and Dominique Lepelley. Some further results on the manipulability of social choice rules. page 28pp, 2004.

[4] Pierre Favardin, Dominique Lepelley, and Jérôme Serais. Borda rule, Copeland method and strategic manipulation. Rev. Econ. Design, 7:213-228, 2002.

[5] Allan Gibbard. Manipulation of voting schemes: a general result. Econometrica, 41:587-601, 1973.

[6] H. C. Huang and Vincent C. H. Chua. Analytical representation of probabilities under the IAC condition. Soc. Choice Welf., 17(1):143-155, 2000.

[7] Jerry S. Kelly. Almost all social choice rules are highly manipulable, but a few aren't. Soc. Choice Welf., 10(2):161-175, 1993.

[8] Dominique Lepelley and Boniface Mbih. The proportion of coalitionally unstable situations under the plurality rule. Econom. Lett., 24(4):311-315, 1987.

[9] Dominique Lepelley and Boniface Mbih. The vulnerability of four social choice functions to coalitional manipulation of preferences. Soc. Choice Welf., 11(3):253-265, 1994.

[10] Dominique Lepelley and Boniface Mbih. Strategic manipulation in committees using the plurality rule: alternative concepts and frequency calculations. Group Decision and Negotiation, 8:21-41, 1999.

[11] Dominique Lepelley and Fabrice Valognes. Voting rules, manipulability and social homogeneity. Public Choice, 116:165-184, 2003.

[12] Shmuel Nitzan. The vulnerability of point-voting schemes to preference variation and strategic manipulation. Public Choice, 47:349-370, 1985.

[13] Geoffrey Pritchard and Arkadii Slinko. On the average minimum size of a manipulating coalition. Soc. Choice Welf., to appear.

[14] Donald G. Saari. Susceptibility to manipulation. Public Choice, 64:21-41, 1990.

[15] Mark Allen Satterthwaite. Strategy-proofness and Arrow's conditions: Existence and correspondence theorems for voting procedures and social welfare functions. J. Econom. Theory, 10(2):187217, 1975. 
[16] Alexander Schrijver. Theory of linear and integer programming. Wiley-Interscience Series in Discrete Mathematics. John Wiley \& Sons Ltd., Chichester, 1986. A Wiley-Interscience Publication.

[17] Arkadii Slinko. On asymptotic strategy-proofness of classical social choice rules. Theory and Decision, 52(4):389-398, 2002.

[18] Arkadii Slinko. How the size of a coalition affects its chances to influence an election. Soc. Choice Welf., to appear.

[19] David A. Smith. Manipulability measures of common social choice functions. Soc. Choice Welf., 16(4):639-661, 1999.

[20] Alan D. Taylor. Social choice and the mathematics of manipulation. Outlooks. Cambridge University Press, Cambridge, 2005. 